\title{
Determinación de la fatiga visual y su relación con el teletrabajo en el personal administrativo de la facultad de Ingeniería de la Universidad Nacional de Chimborazo: Caso práctico
}

\author{
Determination of visual fatigue and its relation to teleworking in \\ administrative personnel of the National University of Chimborazo: Case \\ study
}

Edison Verdezoto Espinoza. ${ }^{1} \&$ Edmundo Cabezas Heredia. ${ }^{2}$

Recibido: 17-07-2021 / Revisado: 30-07-2021 /Aceptado: 21-08-2021/ Publicado: 05-09-2021

DOI: https://doi.org/10.33262/anatomiadigital.v4i3.1.1909

\begin{abstract}
Introduction, according to the survey applied to the administrative staff of the Faculty of Engineering of the National University of Chimborazo, $77.8 \%$ use the computer for more than 4 hours and $22.2 \%$ from 2 to 4 hours. Objective, it is to assess the levels of visual computer syndrome by means of the CVSS 17 test exposed to digital display screens. Methodology, it was carried out through a cross-sectional study applied to 27 administrative staff from the Faculty of Engineering.

Sociodemographic
\end{abstract}

\section{Resumen}

Introducción, según la encuesta aplicada al personal administrativo de la Facultad de Ingeniería de la Universidad Nacional de Chimborazo el $77.8 \%$ usa más de 4 horas el computador y $22.2 \%$ de 2 a 4 horas. Objetivo, es valorar los niveles del síndrome informático visual por medio del test CVSS 17 expuestos a pantallas de visualización digital. Metodología, se realizó mediante un estudio transversal aplicado a 27 administrativos de la Facultad de Ingeniería. Se evaluaron variables sociodemográficas del personal. Se aplicó el test CVSS 17 para medir si existe fatiga

1 Universidad Nacional de Chimborazo, Posgrado, Riobamba, Ecuador, everdezoto@unach.edu.ec ORCID: (iD https://orcid.org/0000-0001-8559-0991

2 Universidad Nacional de Chimborazo, Facultad de Ingeniería, Riobamba-Ecuador, ecabezas@unach.edu.ec ORCID: (D) https://orcid.org/0000-0001-5708-0054 
variables of the personnel were evaluated. The CVSS 17 test was applied to measure whether there is visual fatigue. The reliability of the instrument is 0.87 Results, the results obtained $22.2 \%$ are asymptomatic and $77.8 \%$ have visual fatigue. $22.2 \%$ have moderate level 3 fatigue, $66.7 \%$ moderate level 4 and $11.1 \%$ Severe Level 5. Conclusion, through the use of the test designed to measure the ocular and visual symptoms associated with the use of PVD, $77.8 \%$ of visual fatigue is presented. The presence of clinical alterations has been shown to be significantly associated with telework, a pandemic, the age of the individuals, and previous visual illnesses.

Keywords: telecommuting, Covid 19, administrative staff, CVSS 17. visual. La fiabilidad del instrumento es de 0.87 Resultados, los resultados obtenidos $22.2 \%$ son asintomáticos $\mathrm{y}$ $77.8 \%$ tienen fatiga visual. El $22.2 \%$ tiene fatiga moderada nivel 3, $66.7 \%$ moderado nivel 4 y $11.1 \%$ Severo Nivel 5. Conclusión, mediante el uso del test diseñado para medir los síntomas oculares y visuales asociados al uso de PVD, se presenta un $77.8 \%$ de fatiga visual. La presencia de alteraciones clínicas se ha mostrado significativamente asociada al teletrabajo, pandemia, la edad de los individuos, enfermedades visuales previas.

Palabras claves: teletrabajo, Covid 19, personal administrativo, CVSS 17.

\section{Introducción}

La Organización Mundial de la salud (OMS), declara la presencia del SARS-CoV-2, subsidiario de la enfermedad Covid-19, considerada como pandemia de origen biológico desconocido. Está enfermedad se extiende rápidamente en todos los países del mundo y de manera rápida. Las consecuencias fatales, dificultad para combatir la pandemia por su difícil control (Organización Mundial de la Salud [OMS], 2020). China es el país en donde se originó el virus, pero con el tiempo se ha reducido el número de contagios en este territorio, siendo está controlada se ha reducido el número de casos y el gobierno asegura que la epidemia está controlada. En cambio, el contagio en otros países no ha corrido con la misma suerte ha tardado mucho y aún no se ha controlado en su totalidad a pesar de encontrarse la mayor parte de la población inmunizada y el Ecuador no es la excepción.

La Universidad Johns Hopkins, realizó un seguimiento de los casos en el mundo, el crecimiento en cifras es vertiginoso. La epidemia ha traído consecuencias catastróficas de enfermedad, muerte y pobreza con pérdidas económicas incalculables que afecta a los países del tercer mundo o en vía de desarrollo que viven en un sistema de endeudamiento permanente (Santillán, 2019).

Los departamentos de talento humano continuamente buscan modificar las estructuras de las organizaciones, con criterios de que sean flexibles, horizontales, planas y otros aspectos que permitan una mejora en las empresas y una de esas es el teletrabajo que no 
es nuevo y que tiene una tendencia de futuro por esta nueva realidad. Las empresas, los centros de atención al cliente para llamadas de auxilio o de negocio especializado gestionan desde su casa la acción comercial como herramienta de trabajo (Borda, 2020; Fernández, 2020).

La Organización Internacional del Trabajo (OIT) establece al teletrabajo como una nueva forma de trabajar, lejos del lugar habitual a la casa, con distanciamiento de sus compañeros o del entorno laboral, el teletrabajo integra el uso de las Tics (Tecnologías de la Información y de la Telecomunicación), facilitando la comunicación empresarial para el desarrollo de las diferentes tareas asignadas (Ubieto, 2020).

Existe tareas que se realizan de manera presencial y otras a distancia como es el caso del teletrabajo mediante el uso de los sistemas de tecnología y comunicación (Tics), los tiempos de desplazamiento de la casa a la oficina y viceversa son menores, reducen tiempos muertos por retroceso de acciones en el proceso. El teletrabajo es planificado se plantea metas y objetivos diarios así como las horas dedicadas al trabajo (Bellido, 2006).

El teletrabajo no tiene afección laboral, no vulnera los derechos del trabajador, ni genera terminación laboral con la empresa. El empleador podrá optar por esta modalidad en cualquier instante según la necesidad de la empresa y función del trabajador (Ministerio del Trabajo, 2020).

El teletrabajo en el mundo, sus cambios, el incremento en el uso de las Tics, el aumento de pantallas de visualización digital (PVD) en los centros laborales. Se da una encuesta en Europa sobre las condiciones de trabajo 2015, en el que el $37 \%$ de los trabajadores utiliza PVD en la jornada laboral y $20 \%$ utiliza un cuarto de la jornada día este medio (Eurofund, 2017).

Las Tics se han incorporado a actividades normales como extralaborales. Según Seguí et al, menciona que se debe revisar protocolos de vigilancia de la salud visual de los usuarios de PVD para generar condiciones adecuadas como es el confort visual, al revisar información referente alteraciones óculo-visuales y músculo-esqueléticas asociadas al trabajo con carácter sistemático Molina-Aragones et al (s.f.), manifiesta que se debe mejorar las estrategias para garantizar una vigilancia de la salud eficiente para los usuarios expuestos.

La fatiga visual debido al estado de cansancio de los ojos, provoca enrojecimiento e hinchazón por las jornadas de trabajo demasiado extendidas en PVD y fuentes de iluminación general, localizadas durante largas horas frente a un ordenador u otra fuente de iluminación en exceso o déficit con lo que estipula la normativa (Ramírez, 2014). El trabajo en PVD requiere de iluminación sin brillo, ni deslumbramientos con niveles aceptables entre 300 y 500 lux. Niveles bajo el intervalo se aceptarían con muy poco contraste y niveles superiores aumenta la fatiga visual por lo que es necesario medir los niveles de iluminación (Instituto de Seguridad e Higiene en el trabajo [INSHT], NTP 139). 
Según Bleham et al. (2005), la fatiga visual debida al trabajo por PVD el síndrome óculovisual (SOV) tiene una o varios síntomas visuales (vista cansada, fatiga ocular, ardor, sensibilidad, irritación de los ojos, "ojo rojo", "visión borrosa" y "ojos secos"). Estos factores se atribuyen al síndrome, pero ni se conocen las causa iniciales del problema. Los síntomas con nivel alto por uso de PVD que se reflejan en forma de fatiga visual (Portello, et al, 2012; Kowalska et al, 2011), ardor ocular (Agarwal et al, 2013), alteraciones visuales, lagrimeo, sequedad ocular o dolor de cabeza (Talwar et al, 2009), entre otros.

Los síntomas de la fatiga visual son diferentes no corresponde a una sola enfermedad, sino a diferentes con síntomas parecidos que se relacionan entre sí, muchas de las veces se presentan juntas en una sola persona, la fatiga visual tiene diversidad de orígenes que pueden ser subjetivos, propias de cada persona, tienen variedad en frecuencia e intensidad con características diferentes entre personas a pesar de ser el mismo cuadro clínico (Rodríguez León, 2015). En la figura 1 se aprécialos síntomas de fatiga visual.

\section{Figura 1}

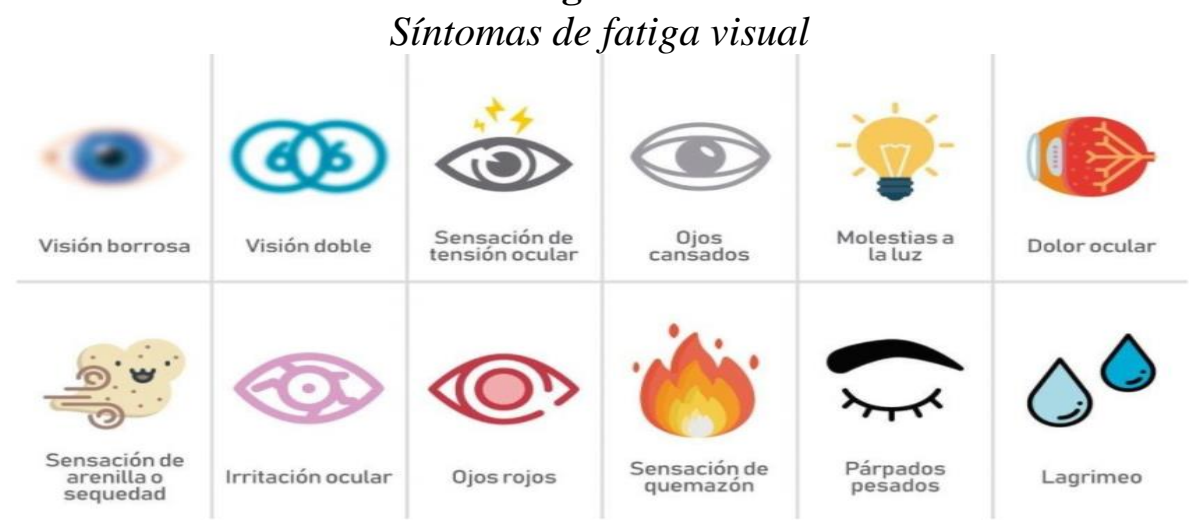

Fuente: Recuperado de https://www.smartoptics.es/es/blog/fatiga-visual-astenopia/

La exposición provoca que la superficie ocular se agrande por la disminución de la frecuencia de los parpados, con evaporación alta lagrimal, mayor sequedad del ojo (Tauste et al., 2014), esto se ve agravado por el uso de lentes de contacto blandos (LC) lo que ayuda a la aparición de sintomatología (González et al., 2007). Al producirse mayor esfuerzo del sistema para acomodar la visión por la mayor frecuencia de exposición a PVD con una distancia corta al ordenador provoca síntomas de fatiga.

En Europa y Asia por el uso de PVD es frecuente el dolor de cuello y nuca (Talwar et al, 2009). El dolor en los discos de las vértebras de la columna, ligamentos, músculos, articulaciones y nervios las causas del dolor son múltiples y pueden incluir tumores, traumas por golpes o fracturas, procesos infecciosos, trastornos inflamatorios o congénitos. Por lo que el uso excesivo de PVD no solo afecta a la parte mental, también a la parte física del trabajador.

La maestría en Prevención de Riesgos Laborales ofertada por la Universidad Nacional de Chimborazo (UNACH) para mejorar en la especialización requerida, plantea que debido al tiempo de exposición que pasa el personal frente a los dispositivos tienden a sufrir daños en el órgano ocular y es por ello que se hace necesaria la evaluación de esta 
sintomatología para poder determinar si el teletrabajo en el personal administrativo tenga incidencia por los contagios de Covid 19 que aún se mantiene, se sigue tomando como alternativa el teletrabajo, por lo cual se realiza la presente investigación en el personal administrativo de la Facultad de Ingeniería de la Universidad Nacional de Chimborazo.

El problema planteado para realizar la investigación y dar solución a la problemática es: ¿La Aplicación del test CVSS 17 permite determinar la fatiga visual y su relación con el teletrabajo en el Personal administrativo de la Facultad de Ingeniería de la Universidad Nacional de Chimborazo?

\section{Metodología}

El estudio fue de tipo cuantitativo, con diseño transversal en un solo instante de tiempo para la recolección de datos y análisis, estudio observacional, descriptivo del fenómeno tal como se presenta, los análisis se realizaron a través de un enfoque estadístico univariado y cruce de variables por lo que es correlacional. La población fue seleccionada de todo el personal administrativo de la Facultad de Ingeniería de la Universidad Nacional de Chimborazo se conformó por 27 personas, no existe muestreo se trabaja con toda la población.

Los criterios de inclusión fueron:

$\checkmark$ Trabajadores con diferentes tipos de acción de personal.

$\checkmark$ Trabajadores que se encontraran realizando teletrabajo.

$\checkmark$ Trabajadores que estuvieron de acuerdo con el consentimiento informado.

$\checkmark$ Trabajadores con exposición al computador como mínimo 4 horas al día y máximo 20 horas semanales.

La recolección de los datos se realizó con el test creado en Google forms y difundido el link al personal de administrativo de la Facultad de Ingeniería de la Universidad Nacional de Chimborazo, la data recolectada en Excel se exporta al SPSS V24 para su programación y obtención de resultados. Se estableció variables sociodemográficas para determinar correlaciones con el test del Síndrome. Se determinó el Alpha de Cronbach para medir la fiabilidad para el cuestionario de 0.87 , lo cual indica una consistencia interna elevada para determinar la fiabilidad y confiabilidad del test (Huapaya, 2019).

El cuestionario consta con 17 ítems. Cada respuesta se valora con una puntuación en un formato de escala según lo contestado por el trabajador en cada pregunta realizada. Se describen el número de opciones por cada pregunta a continuación:

$\checkmark 10$ preguntas con cuatro opciones de respuesta: nunca, raramente, frecuentemente y constantemente.

$\checkmark 6$ preguntas con opciones de respuesta: nada, si muy poco, si un poco, si moderadamente, si mucho, si muchísimo.

$\checkmark 1$ pregunta con opción de respuesta: nunca, casi nunca, poco tiempo, parte del tiempo, mucho tiempo, casi siempre, siempre. 
Los puntajes de respuesta para determinar el nivel de sintomatología del personal administrativo de la Facultad de Ingeniería se lo realizó con la ecuación: CVSS17 = (Suma puntuación total de todas las preguntas).

La puntuación entre 17 y 53 puntos; o con puntajes mayores la sintomatología es evidente del síndrome, con puntajes igual o mayor a 36 se lo considerará sujeto sintomático para fatiga visual y valores menor a 36 será relacionado como asintomático (Arlanzón Lope, 2018).

El cuestionario CVSS 17 es una herramienta de fácil acceso, que puede ser manejado para la vigilancia médica por cualquier especialidad de salud que haya sido con anterioridad capacitado en el contexto de seguridad e higiene de trabajo, su factibilidad en la interpretación y su disponibilidad en diferentes idiomas favorece un proceso de adaptación cultural y aceptación con rangos de fiabilidad que demuestran consistencia interna asegurando su validación (Huapaya, 2019).

Existen niveles del Síndrome los mismos que van desde 1 a 6 estableciendo el nivel de gravedad desde leve a severo como se detalla a continuación: Leve: Nivel 1 puntaje de 17 a 22; Nivel 2 puntaje de 23 a 28; Moderado: Nivel 3 puntaje de 29 a 35; Nivel 4 puntaje de 36 a 42; Severo: Nivel 5 puntaje de 43 a 49; Nivel 6 puntaje de 50 a 53

\section{Resultados y discusión}

Los resultados de aplicar el test CVSS 17 en el personal administrativo de la Facultad de Ingeniería de la Universidad Nacional de Chimborazo son los siguientes:

En la tabla 1 se presenta el género del personal administrativo de la Facultad de Ingeniería de la UNACH.

Tabla 1

Género del Personal administrativo de la Facultad de Ingeniería de la UNACH

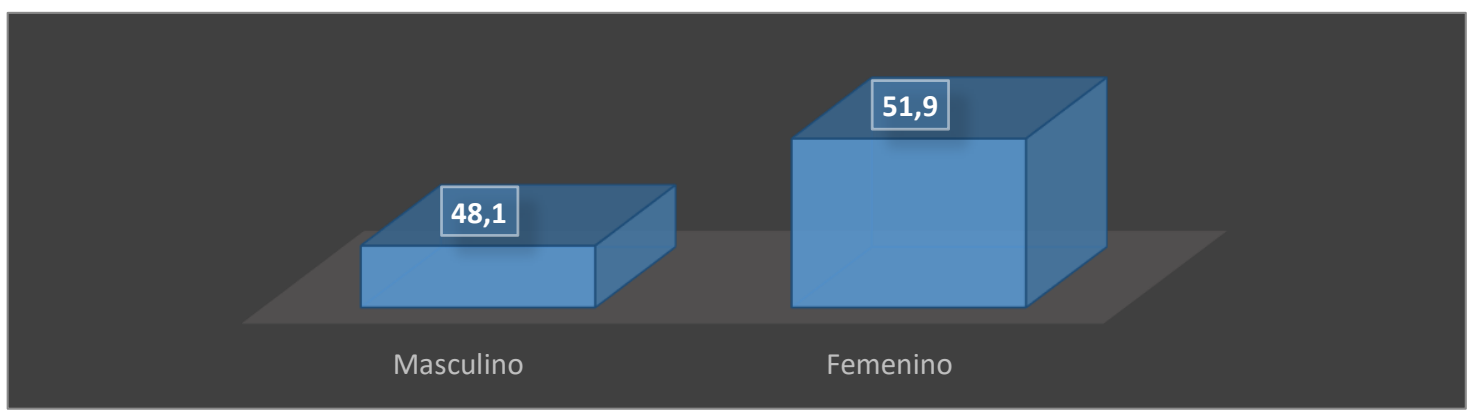

Referente al género del personal administrativo de la Facultad de Ingeniería de la Universidad Nacional de Chimborazo se tiene: $51.9 \%$ son mujeres y el $48.1 \%$ hombres por lo que se puede determinar que existe equilibrio de género en el personal.

En la tabla 2 se presenta el nivel educativo del personal administrativo de la Facultad de Ingeniería de la UNACH. 


\section{Tabla 2}

Nivel de Educación del Personal administrativo de la Facultad de Ingeniería de la UNACH

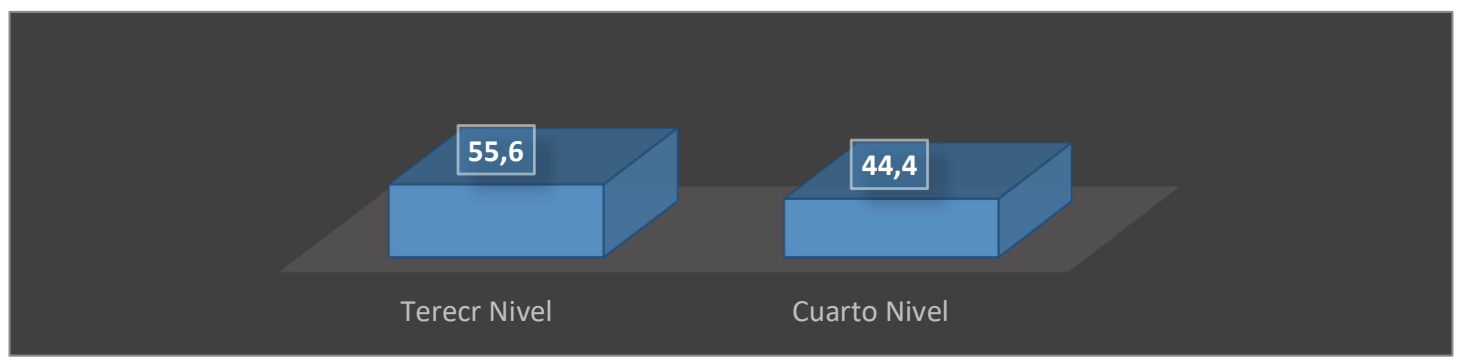

Referente al nivel educativo del personal administrativo de la Facultad de Ingeniería de la Universidad Nacional de Chimborazo se tiene: el 55.6\% tienen tercer nivel y el $44.4 \%$ cuarto nivel por lo que se puede determinar que el personal administrativo cuenta con un nivel de preparación elevado.

En la tabla 3 se presenta el tipo de relación laboral del personal administrativo de la Facultad de Ingeniería de la UNACH.

\section{Tabla 3}

Relación Laboral del Personal administrativo de la Facultad de Ingeniería de la UNACH

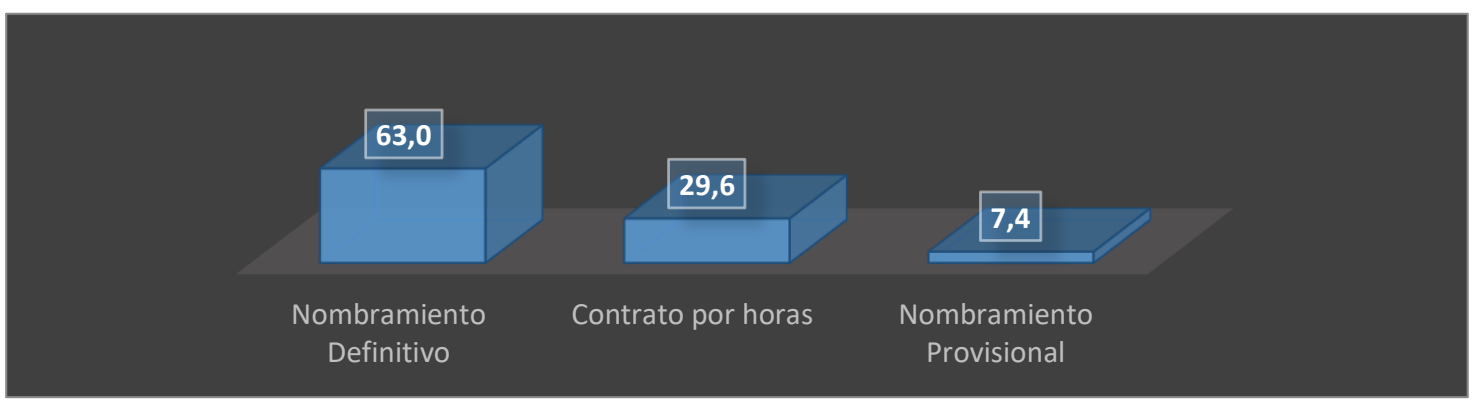

Referente al tipo de relación laboral del personal administrativo de la Facultad de Ingeniería de la UNACH tenemos: el $63 \%$ tienen nombramiento definitivo, $29.6 \%$ contrato por horas y $7.4 \%$ con nombramiento provisional. Se puede determinar que existe una vinculación definitiva con la universidad que motiva al personal.

En la tabla 4 se presenta la edad del personal administrativo de la Facultad de Ingeniería de la UNACH.

\section{Tabla 4}

Edad del Personal administrativo de la Facultad de Ingeniería de la UNACH

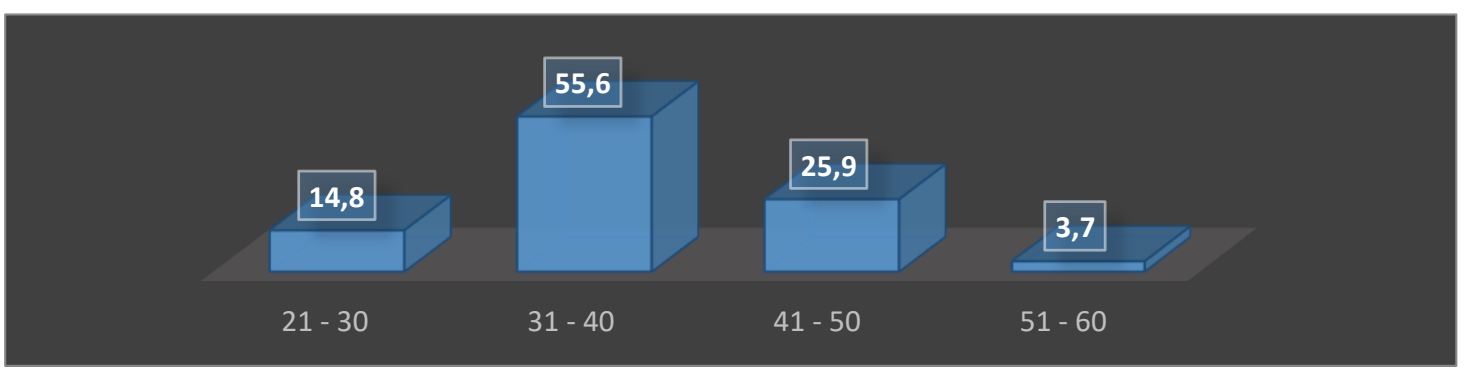


Referente a la edad del personal administrativo de la Facultad de Ingeniería de la Universidad tenemos: $14.8 \%$ tiene entre 21 a 30 años, $55.6 \%$ tiene 31 a 40 años, $25.9 \%$ tiene de 41 a 50 años y $3.7 \%$ de 51 a 60 años siendo relativamente joven el personal.

En la tabla 5 se presenta si el personal administrativo de la Facultad de Ingeniería de la UNACH ha tenido una enfermedad crónica visual.

\section{Tabla 5}

Enfermedad Crónica Visual del Personal administrativo de la Facultad de Ingeniería de la UNACH

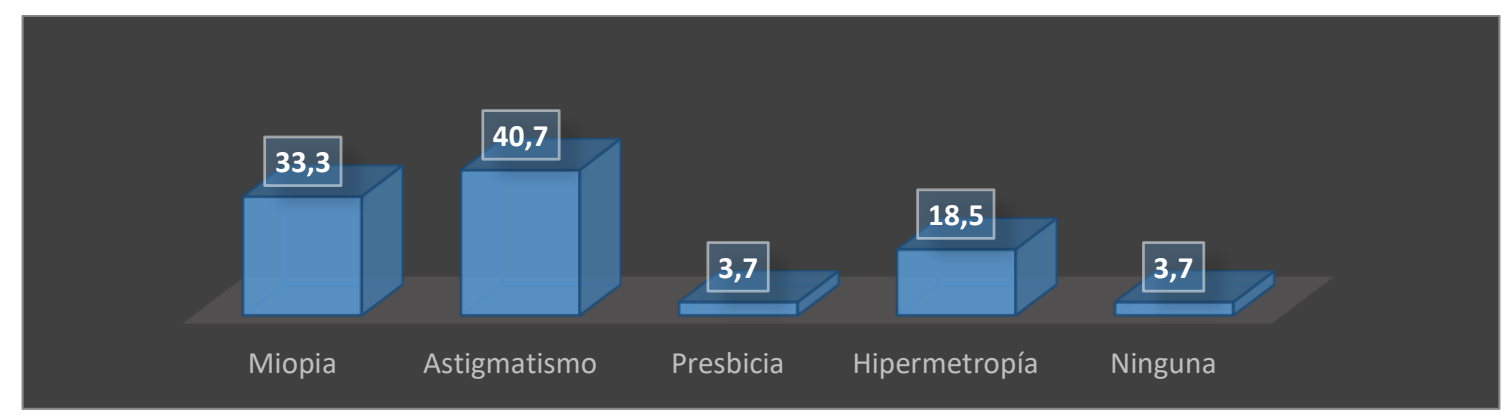

Referente a que el personal administrativo de la Facultad de Ingeniería de la UNACH, si tiene alguna enfermedad crónica visual tenemos: el $33.3 \%$ tiene miopía, el $40.7 \%$ tiene astigmatismo, $3.7 \%$ tiene presbicia, $18.5 \%$ hipermetropía y $3.7 \%$ no tiene ninguna enfermedad lo que permite concluir que estas enfermedades visuales pueden agravarse por uso de pantallas de visualización digital y presentar presencia del síndrome.

En la tabla 6 se presenta el número de horas frente al computador del personal administrativo de la Facultad de Ingeniería de la UNACH.

\section{Tabla 6}

Horas frente al computador del personal administrativo de la Facultad de Ingeniería de la UNACH

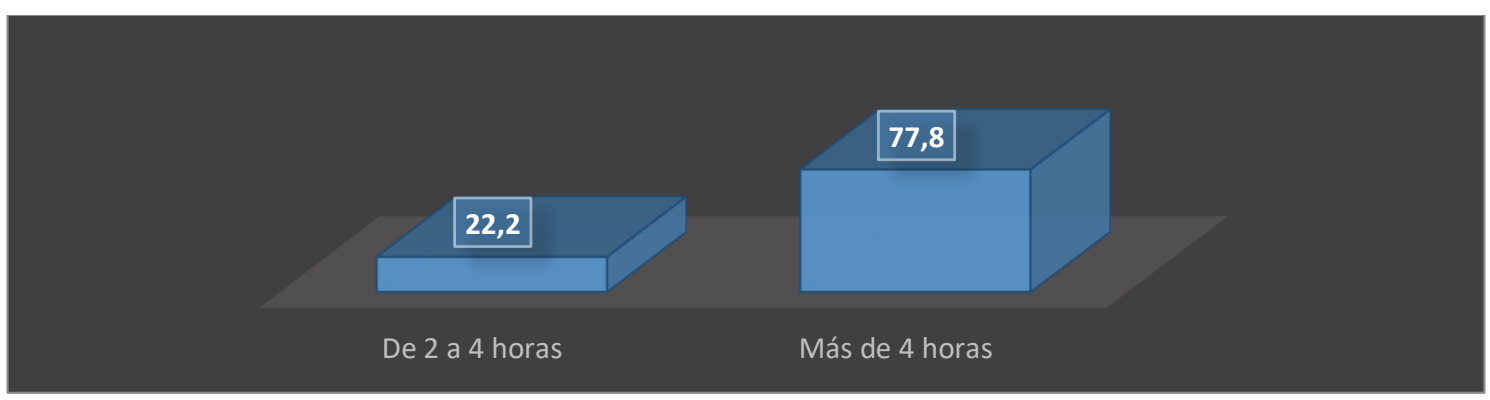

Referente al número de horas frente al computador el personal administrativo de la Facultad de Ingeniería de la Universidad tenemos: $22.2 \%$ de 2 a 4 horas usa el computador y $77.8 \%$ usa más de 4 horas por lo que puede ser propensa a tener el síndrome del computador.

En la tabla 7 se presenta los niveles del síndrome CVSS 17 del personal administrativo de la Facultad de Ingeniería de la UNACH. 


\section{Tabla 7}

Niveles del síndrome CVSS 17 del Personal administrativo de la Facultad de Ingeniería de la UNACH

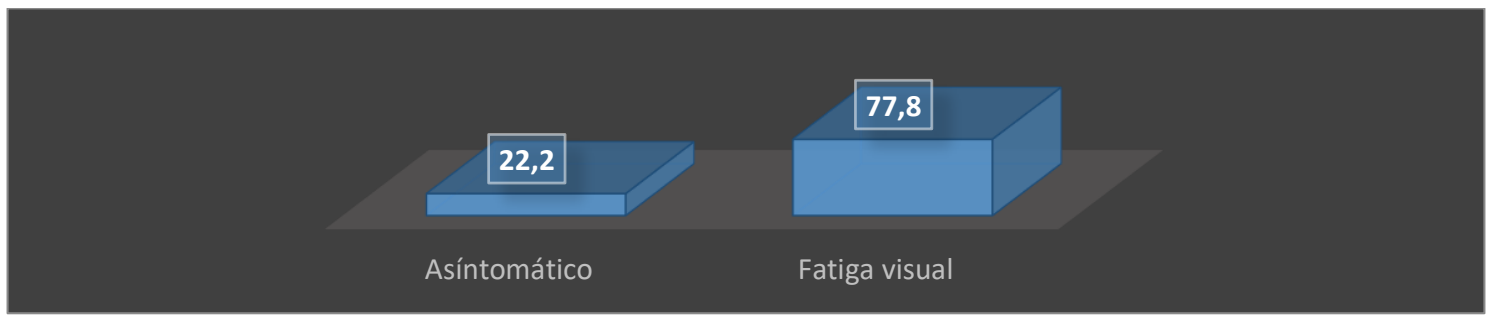

Referente a los niveles del síndrome CVSS 17: el 22.2 \% es asintomático y el $77.8 \%$ tiene fatiga visual por el uso de pantallas de visualización digital por lo que se recomienda tratamiento con un oftalmólogo para prevenir pérdida de visión.

En la tabla 8 se presenta los niveles del síndrome CVSS 17 por tipo del personal administrativo de la Facultad de Ingeniería de la UNACH.

\section{Tabla 8}

Niveles del síndrome CVSS 17 por tipo del personal administrativo de la Facultad de Ingeniería de la UNACH

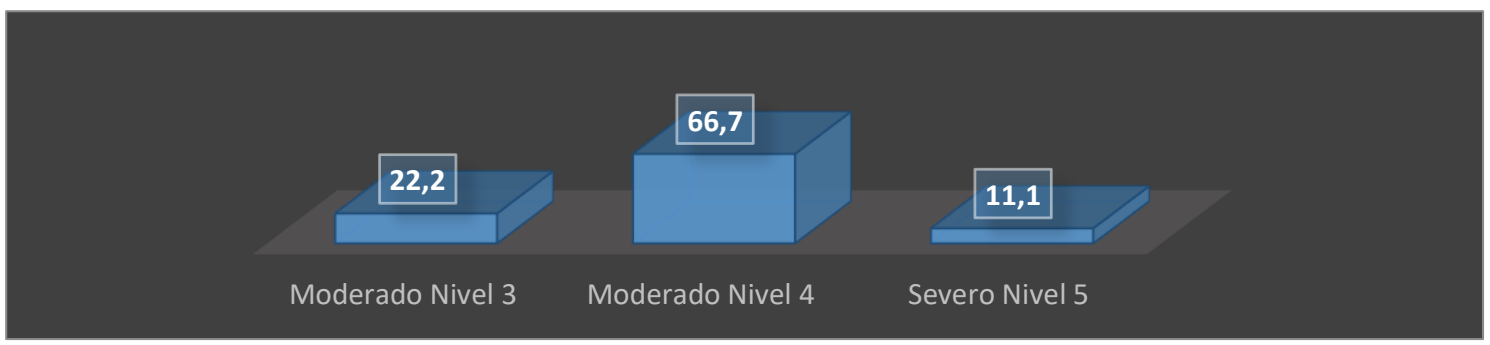

Referente a los niveles del síndrome CVSS 17 de acuerdo al tipo tenemos: el $22.2 \%$ tiene fatiga moderada nivel 3, 66.7 \% moderada nivel 4y $11.1 \%$ severo nivel 5 por lo que existe presencia de fatiga ocular es necesario visitar al oftalmólogo para tener un diagnóstico y reducir la exposición frente al computador.

En la tabla 9 se presenta la correlación entre el género y el Síndrome CVSS 17 del personal administrativo de la Facultad de Ingeniería de la UNACH.

\section{Tabla 9}

Correlación entre Género y el síndrome CVSS 17 del Personal administrativo de la Facultad de Ingeniería de la UNACH

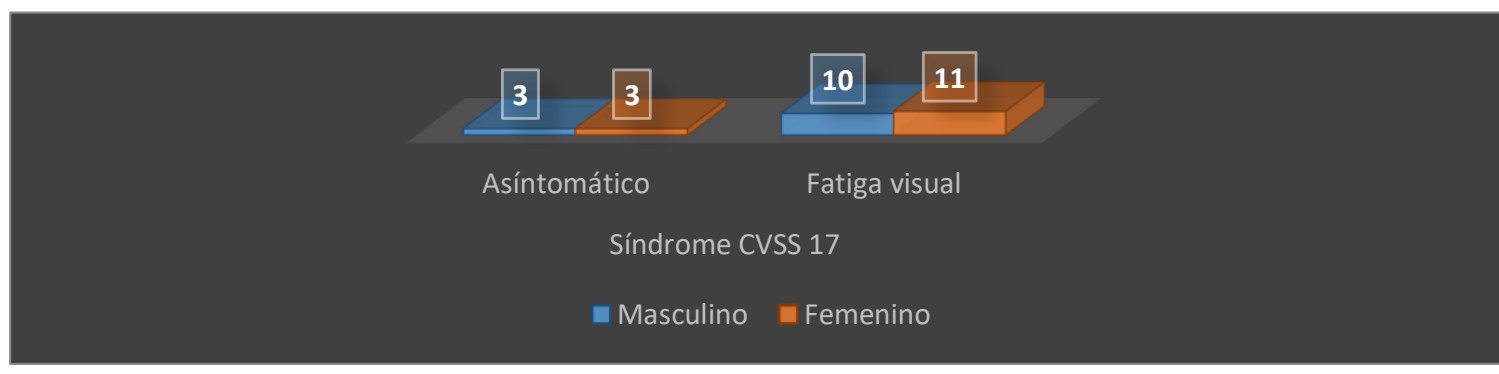




\section{ADDigigital}

ISSN: 2697-3391

www.anatomiadigital.org

Vol. 4, N³.1, p. 149-162, septiembre, 2021

La correlación entre el género y los Niveles del Síndrome por medio del V de Cramer es de 0.020 la cual es baja y no están relacionadas, se tiene que referente a la fatiga visual tienen: 10 hombres y 11 mujeres y 3 hombres y 3 mujeres son asintomáticos.

En la tabla 10 se presenta la correlación entre el nivel de educación y el nivel del Síndrome CVSS 17 del personal administrativo de la Facultad de Ingeniería de la UNACH.

\section{Tabla 10}

Correlación entre nivel de educación y el nivel del síndrome CVSS 17 del Personal administrativo de la Facultad de Ingeniería de la UNACH

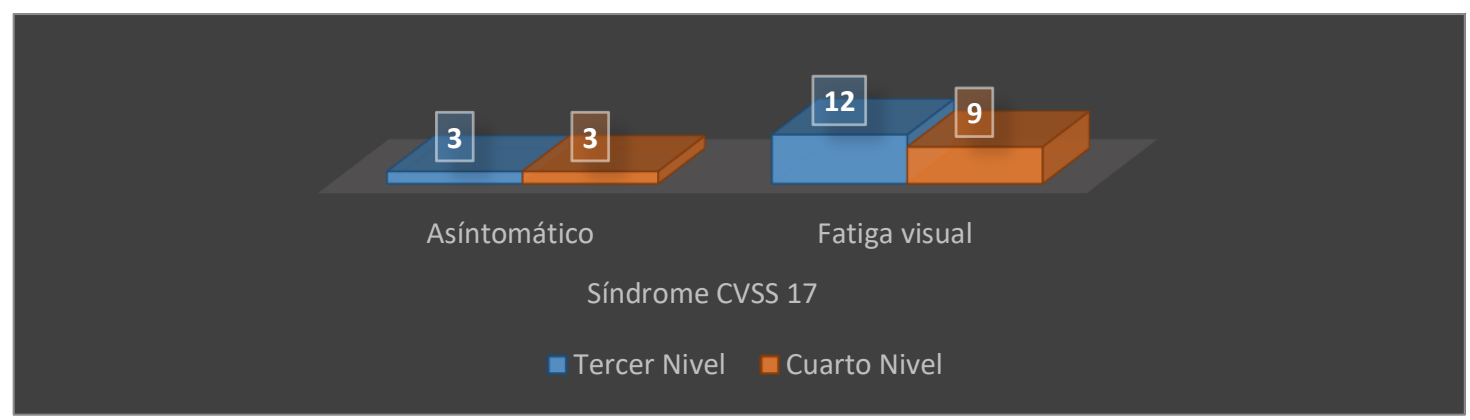

La correlación entre el nivel educativo y el nivel del síndrome CVSS 17 por medio del V de Cramer es de 0.060 es demasiado baja no están relacionadas, se tiene: 3 personas con tercer nivel y 3 de cuarto nivel que son asintomáticos y 12 personas de tercer nivel y 9 de cuarto nivel que tienen fatiga visual, por lo que es necesario medidas preventivas $\mathrm{y}$ vigilancia de la salud en el personal.

En la tabla 11 se presenta la correlación entre horas frente al computador y el nivel del Síndrome CVSS 17 del personal administrativo de la Facultad de Ingeniería de la UNACH.

\section{Tabla 11}

Correlación entre horas frente al computador y el nivel del síndrome CVSS 17 del Personal administrativo de la Facultad de Ingeniería de la UNACH

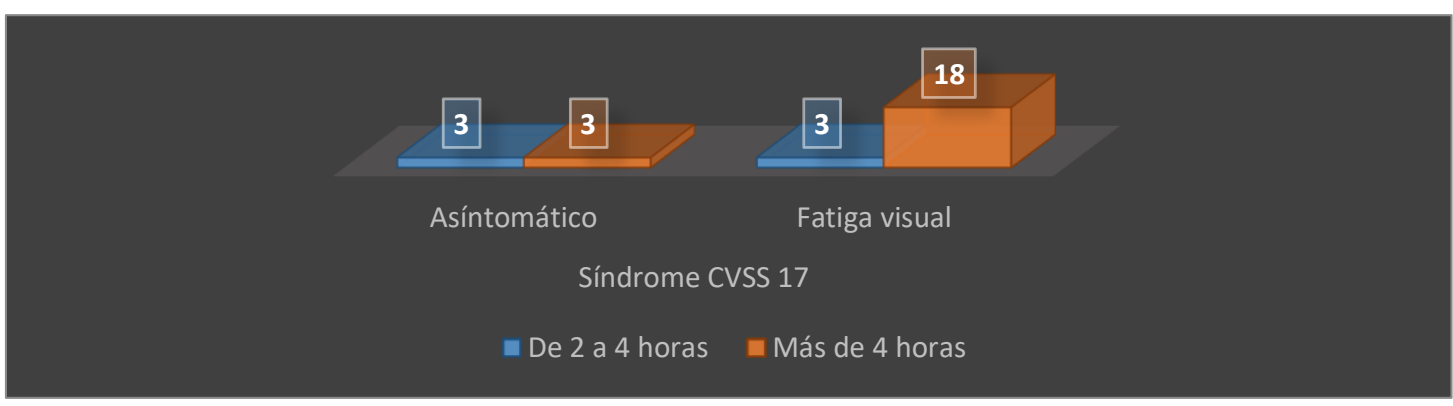

Referente a la correlación entre horas frente al computar y los niveles del síndrome CVSS 17 en el personal administrativo de la Facultad de Ingeniería de la Universidad Nacional de Chimborazo por medio del V de Cramer tenemos: 0.378 existe una relación baja entre las variables, existen 3 personas asintomáticas que pasan frente al computador de 2 a 4 horas, 3 personas asintomáticas que pasan más de 4 horas frente al computador, 3 
personas con fatiga visual que permanecen 2 a 4 horas frente al computador y 18 personas con fatiga visual que permanecen más de 4 horas frente al computador.

En la tabla 12 se presenta la correlación entre edad y el nivel del Síndrome CVSS 17 del personal administrativo de la Facultad de Ingeniería de la UNACH.

\section{Tabla 12}

Correlación entre edad y el nivel del síndrome CVSS 17 del Personal administrativo de la Facultad de Ingeniería de la UNACH

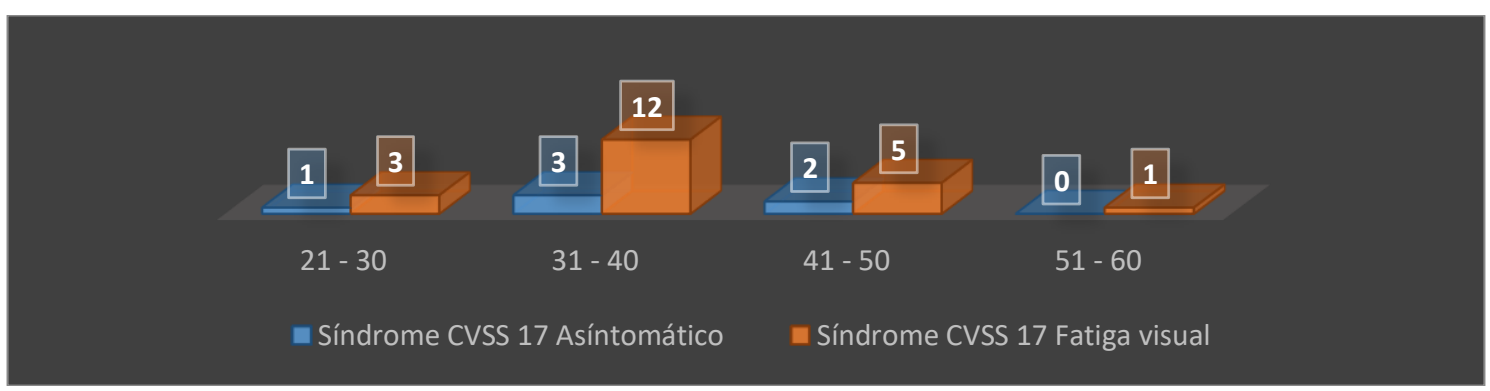

Referente a la correlación entre la edad y el nivel del Síndrome CVSS 17 en el personal administrativo de la Facultad de Ingeniería de la Universidad Nacional de Chimborazo por medio de $V$ de Cramer se tiene: 0.137 es baja la relación, 1 persona de 21 a 30 años es asintomático, 3 personas de 21 a 30 años tiene fatiga visual, 3 personas son asintomáticas de 31 a 40 años, 12 personas tienen fatiga visual de 31 a 40 años, 2 personas asintomáticas de 41 a 50 años, 5 personas tienen fatiga visual de 41 a 50 años y 1 persona tiene fatiga visual de 51 a 60 años.

\section{Conclusiones}

- Los síntomas visuales del CVS diagnóstica en el personal administrativo problemas de visión y de acomodo por demandas exigentes de visión para prevenir y tratarlas se debe tener hábitos adecuados en el trabajo con el computador como buena postura ergonómica, iluminación adecuada que genere confort cuando se usa las pantallas de visualización digital.

- El síndrome de ojo seco es el síntoma ocular de CVS causa sequedad en el ojo por factores de ambiente que llevan a la evaporación excesiva de lágrimas, en el caso de la investigación se detectó que el $22.2 \%$ es asintomático y el $77.8 \%$ tiene fatiga visual por el uso de pantallas de visualización digital.

- La correlación entre las variables sociodemográficas con respecto a los niveles del síndrome CVSS 17 es baja no tiene relación alguna por lo que la presencia del síndrome se debe a otras causales como el teletrabajo, la pandemia y la excesiva frecuencia de exposición a un computador.

- Referente a los niveles por tipo se tiene que el $22.2 \%$ tiene fatiga moderada nivel $3,66.7 \%$ moderada nivel 4 y $11.1 \%$ Severo nivel 5 por lo que existe presencia de fatiga ocular y es necesario visitar al oftalmólogo para tener un diagnóstico, reducir la exposición frente al computador como una medida administrativa. 


\section{Referencias bibliográficas}

Agarwal S. Goel D. Sharma A. Evaluation of the factors which contribute to the ocular complaints in computer users. J Clin Diagn Res. 2013; 7: 331-335.

Arlanzón Lope, P. (2018). Evaluación y caracterización del síndrome visual informático en la población de la Universidad de Valladolid. Valladolid.

Bleham C. Vishnu S. Khattak A. Mitra S. Yee RW. Computer vision syndrome: a review. Surv Ophthalmol.2005;

$50: 253-262$.

Bellido Alexis. Teletrabajo hoy: como hacer dinero y mejorar tu vida trabajando en línea: una guía práctica, 2006.

Borda Díaz, J. ¿Por el coronavirus puedes pedir teletrabajo, cómo gestionarlo? https://www.elempleo.com/co/noticias/noticias-laborales/por-el-coronaviruspuedespedir-teletrabajo -cómo-gestionarlo-6033, 2020.

European Foundation for the improvement of living and Working Conditions (Eurofund). Sixth European Working Conditions Survey. (Consultado 16 de Enero de 2017). Disponible en: https://www.eurofound. europa.eu/publications/report/2016/working-conditions/sixth-european-workingconditions-surveyoverview-report

Fernández, A. El mundo hace frente a la pandemia del Covid-19. Barcelona España: https: //www.lavanguardia.com/autores/alba-fernandez.html, 2020.

Huapaya Caña, Y. A. (2019). Huapaya Caña, Y. A. (2020). Validación del instrumento "Computer Vision Syndrome Questionnaire (CVS-Q)" en el personal administrativo. Lima.

González-Méijome JM, Parafita MA, Yebra-Pimentel E, Almeida JB. Symptoms in a population of contact lens and noncontact lens wearers under different environmental conditions. Optom Vis Sci. 2007; 84:296-302

Instituto de Seguridad e Higiene en el trabajo (INSHT). (1077). El trabajo con pantallas de visualización. https://www.insst.es/documents/94886/326853/ntp_139.pdf/d432d6f6-cbba$\underline{4 a 12-8615-01 \text { eefce } 6865 \mathrm{e} \text { ?version }=1.0 \& \mathrm{t}=1617977205796}$

Kowalska M. Zejda JE. Bugajska J. Braczkowska B. Brożek G. Malińska M. Eye simptoms in office employees working at computer stations. Med Pr. 2011; 62: 18

Ministerio de Trabajo del Ecuador. (2020). Acuerdo Ministerial Nro. MDT - 2030 - 181 Quito: Ministerio de Trabajo. 
Molina-Aragonés JM. Forns-Carbonell J. Rodríguez-Moreno JM. Sol-Vidiella J. LópezPérez C. Revisión sistemática sobre las alteraciones óculo-visuales y músculoesqueléticas asociadas al trabajo con pantallas de visualización de datos. Med Segur Trab (Internet). 2017. 63(247): 159-197.

Organización Mundial de la Salud [OMS]. (2020, Abril 27). Retrieved from Enfermedad del COVID 19: https://www.who.int/es/emergencies/diseases/novelcoronavirus2019/advice-for-public/q-a-coronaviruse,

Portello JK. Rosenfield M. Bababekova Y. Estrada JM. León A. Computer-related visual symptoms in office workers. Ophthalmic Physiol Opt. 2012; 32: 375-382.

Ramírez Vázuez, H. (2014). Bienestar y Calidad de Vida, Medicina del trabajo.

Rodríguez León, A. (2015). Salud ocupacional en optometría: importancia de la ergonomía y prevención visual para mejorar el rendimiento en áreas industriales. Guayaquil.

Sa EC. Junior MF. Rocha LE. Risk factors for computer visual síndrome (CVS) among operators of two call centers in São Paulo, Brazil. Work. 2012; 41: 3568-3574

Santillán, W. La era del talentismo, el gran reto de los gestores humanos en las organizaciones. Quito - Ecuador: Don Bosco, 2019.

Seguí Crespo MM. Ronda Pérez E. López Navarro A. Juan Pérez PV. Tascón Bernabéu E. Martínez Verdú FM. Protocolo de vigilancia sanitaria de trabajadores con pantallas de visualización de datos: una valoración desde la perspectiva de la salud visual. Rev. Esp Salud Pública 2008; 82: 691-701

Talwar R. Kapoor R. Puri K. Bansal K. Singh S. A study of visual and musculoskeletal health disorders among computer professionals in NCR Delhi. Indian J Community Med. 2009; 34: 326-328.

Tauste Francés A, Ronda-Pérez E, Del Mar Seguí Crespo M. Alteraciones oculares y visuales en personas que trabajan con ordenador y son usuarias de lentes de contacto. Una revisión bibliográfica. Rev. Esp Salud Pública 2014; 88:203-215.

Ubieto, G. El teletrabajo una "nueva normalidad" para unos pocos, https://www.elperiodico.com/es/economia/20200502/el-teletrabajo-unanuevanormalidad-para-unos-pocos-7947524, 2020.

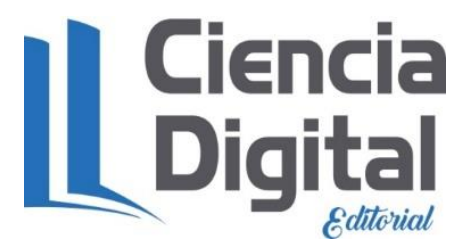




\section{PARA CITAR EL ARTÍCULO INDEXADO.}

Verdezoto Espinoza, E., \& Cabezas Heredia, E. (2021). Determinación de la fatiga visual y su relación con el teletrabajo en el personal administrativo de la facultad de Ingeniería de la Universidad Nacional de Chimborazo: Caso práctico . Anatomía Digital, 4(3.1), 149-162. https://doi.org/10.33262/anatomiadigital.v4i3.1.1909

\section{Liencia}

El artículo que se publica es de exclusiva responsabilidad de los autores y no necesariamente reflejan el pensamiento de la Revista Anatomía Digital.

El artículo queda en propiedad de la revista y, por tanto, su publicación parcial y/o total en otro medio tiene que ser autorizado por el director de la Revista Anatomía Digital.
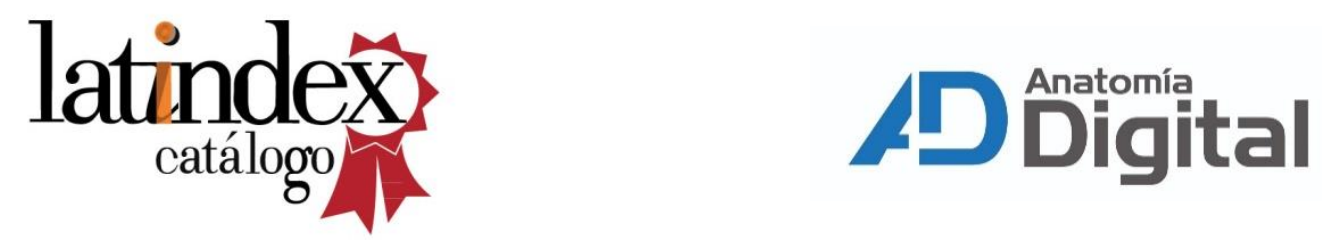